\title{
Emergency and urgent dental visits among Medicaid enrollees from 2013 to 2017
}

\author{
Rebekah Fiehn¹, llya Okunev ${ }^{1}$, Mary Bayham¹', Steven Barefoot ${ }^{2}$ and Eric P. Tranby ${ }^{1 *}$ (C)
}

\begin{abstract}
Background: Better understanding of the frequency of dental emergencies and the procedures performed during those emergency visits can help providers, insurers, and policymakers understand workforce and care provision needs.

Methods: Procedures performed at an emergency dental encounter and in the encounter following that encounter are assessed. Emergency dental encounters are those with a CDT code of D0140, D0160, or D0170. Data was analyzed from the IBM Watson Medicaid Marketscan data from 2013 to 2017, a nationally representative dental and medical claims database from 13 deidentified states in the United States.
\end{abstract}

Result: Consistently over time, about $10 \%$ of all dental encounters are due to a dental emergency. $28 \%$ of emergency dental encounters had no other procedure performed during those encounters. When other procedures were performed during the encounter, the majority were diagnostic in nature, primarily radiographs. Among patients who returned to the dentists following an emergency visit, $43 \%$ returned for more definitive dental treatment, most within 30 days.

Conclusions: The majority of dental emergency encounters do not result in definitive treatment, rather patients often return to the dentist at a later date for that treatment. Where possible, dental providers could utilize teledental services to triage patients to appropriate care.

Keywords: Emergency dental, COVID-19, Teledental, Medicaid

\section{Background}

It is important for health care systems to have a general understanding of what constitutes a dental emergency to ensure that patients have access to essential care and establish best practice pathways for determining appropriate care location. Providers need to understand the critical elements of administering emergency dental care, the factors which may influence patients to seek out care, and plan appropriately for triaging and treating emergency cases. However, there is a gap in research around dental emergencies in dental settings. The research

*Correspondence: Eric.Tranby@dentaquest.com

1 DentaQuest Partnership for Oral Health Advancement, 465 Medford

Street, Boston, MA 02129, USA

Full list of author information is available at the end of the article available in the United States has largely focused on pediatric dental emergencies including those originating from trauma [1, 2]. A study out of South Carolina found that just $9 \%$ of the after-hours pediatric dental emergencies analyzed needed referral to ED for treatment while the rest could be addressed in the dental setting. Additionally, the study found that there was significant variation in the treatment decisions partly due to unique provider characteristics (pediatric vs general) or practice settings [1]. Studies from outside of the US have mainly focused on the reasons for the emergency dental visit, with little emphasis on what happens following the emergency dental visit $[3,4]$.

In the United States, limited access and reductions in covered services by public health programs often lead to increases in emergency department (ED) services. A 
study of the emergency department visits at the University of Illinois Hospital found the reduction in dental benefits was followed by increases in ED visits (48\%), surgical interventions (100\%), and hospital admission days (128\%) [5]. Most dental care in the ED is palliative and consists of infection management through antibiotics and pain management through analgesics. Most EDs are not equipped to provide definitive care for dental conditions such as dental pulpal or periapical lesions, cellulitis or abscess, injuries, and pain. ED interventions are directed toward treating symptoms of the underlying condition without resolving the primary issue which often leads to revisits and may lead to the over prescribing of opioids and antibiotics [6-12]. ED resources and care teams should be focused on the management of infectious and critically ill patients, so it is vital that dental emergencies are kept in dental settings where appropriate and definitive treatment can be established.

Better understanding of the frequency of dental emergencies and the procedures performed during emergency visits can help providers, insurers, and policymakers understand workforce and care provision needs both within and outside of the coronavirus disease 2019 (COVID-19) pandemic environment. To that end, a retrospective study of data of Medicaid claims from 2013 through 2017 is used to (1) identify trends in emergency dental visits, (2) describe what happens during emergency dental visits, and (3) identify common treatment pathways following emergency dental visits.

\section{Methods}

This retrospective study used Medicaid claims data to study the prevalence and composition of Emergency Dental visits.

\section{Data source}

This study used deidentified medical, dental, and pharmaceutical claims data from January 1, 2013 through December 31, 2017 from the IBM Watson MarketScan Multi-State Medicaid Database core data set [13]. This is a very large database with billions of records on millions of patients, has been used in hundreds of peer reviewed publications, and is generally accepted as a nationally representative dental and medical claims database of Medicaid data. That said, the data includes all Medicaid claims from 13 deidentified states and, therefore, are not drawn from a random sample. As such, findings are not guaranteed to generalize to the larger U.S. population [14].

\section{Sample selection}

Emergency dental encounters are those with a Code on Dental Procedures and Nomenclature (CDT) code of D0140, D0160, or D0170, which are codes commonly used to identify limited exams (D0140), extensive (D0160) exams, or re-evaluations (D0170) done by a dentist in a dentist's office or similar facility on an urgent or emergency basis. CDT codes are the standard set of procedural codes for oral health, commonly used for billing purposes, and widely available in claims data. Because the findings show consistency in trends over time, we often focus on a single year analysis, which is typically 2016 . Procedures performed at an emergency dental encounter in 2016 are assessed, and are sometimes compared to procedures performed at non-emergency dental encounters in the same year. Follow-up encounters occurring between 1 and 365 days after the initial emergency dental encounter are also assessed. Follow-up encounters may occur at any time in 2016 or 2017.

We included all patients aged 0 to 64 with at least 1 dental claim in the year 2016 in the study. In line with Medicaid policy, patients aged 0 to 20 are defined as children. Patients aged 21 to 64 are defined as adults. These categories are based on eligibility for child versus adult Medicaid.

\section{Variables}

We defined prescriptions as being associated with an emergency dental visit if the prescription started on the same day as an emergency dental visit, regardless of whether patients had other visits or not that day.

When comparing emergency dental visits to follow-up visits, the first emergency dental visit in the year for the patient is considered as the index emergency visit. The follow-up visit by the patient may be another emergency dental visit, a treatment visit or a routine office visit. A treatment visit in this case is defined by procedure codes D2XXX-D7999, which includes restorative procedures, oral surgeries, and endodontic procedures, while a routine office visit is considered to be any non-emergency, non-treatment visits to a dentist, incorporating routine examination, diagnostic services, and preventive care.

In some parts of the analysis, we used the integrated nature of the claims database and the detailed information on the timing of visits to examine the correlation between emergency dental encounters in the dental office and visits to the hospital emergency room (ER) for dental conditions. Dental conditions are defined using the ASTDD classification of ICD-10 diagnostic codes for non-traumatic dental conditions We examined ER visits that occurred within 15 days of both emergency and nonemergency dental visits (visits that occurred at a dentist's office). We are also examined the number of emergency dental and ER visits that occurred within 1 year of the emergency dental visit. 
Table 1 Demographic characteristics of study population (in 2016)

\begin{tabular}{|c|c|c|c|c|c|}
\hline & Variable & $\begin{array}{l}\text { Medicaid enrolled } \\
\text { population (IBM Watson } \\
\text { Data) }\end{array}$ & $\begin{array}{l}\text { Nationally representative } \\
\text { sample of Medicaid enrolled } \\
\text { population }\end{array}$ & $\begin{array}{l}\text { Population with at least } \\
\text { one dental visit (IBM } \\
\text { Watson) }\end{array}$ & $\begin{array}{l}\text { Population with one or more } \\
\text { emergency dental visits (IBM } \\
\text { Watson) }\end{array}$ \\
\hline & Count & $\%$ within categories & $\%$ within categories & $\%$ within categories & $\%$ within categories \\
\hline \multicolumn{6}{|l|}{ Sex } \\
\hline Male & $5,721,884$ & 43 & 44 & 46 & 39 \\
\hline Female & $7,571,087$ & 57 & 56 & 54 & 61 \\
\hline \multicolumn{6}{|l|}{ Race } \\
\hline White & $6,436,589$ & 47 & 40 & 47 & 51 \\
\hline Black & $4,245,692$ & 32 & 19 & 32 & 30 \\
\hline Hispanic & 704,674 & 9 & 29 & 9 & 6 \\
\hline Other & $1,906,016$ & & 12 & 12 & 13 \\
\hline \multicolumn{6}{|l|}{ Age } \\
\hline $0-10$ & $4,054,204$ & & 30 & 46 & 29 \\
\hline $11-20$ & $2,955,882$ & & 25 & 32 & 24 \\
\hline $21-34$ & $2,220,470$ & & 18 & 10 & 21 \\
\hline $35-51$ & $1,684,552$ & & 15 & 8 & 16 \\
\hline $51-64$ & $1,329,492$ & & 12 & 5 & 11 \\
\hline Total & $12,244,600$ & 100 & 100 & 32 had dental visit & 5 had emergency dental visit \\
\hline
\end{tabular}

National estimates of the Medicaid enrolled population are derived from the Medical Expenditure Panel Survey (2016)

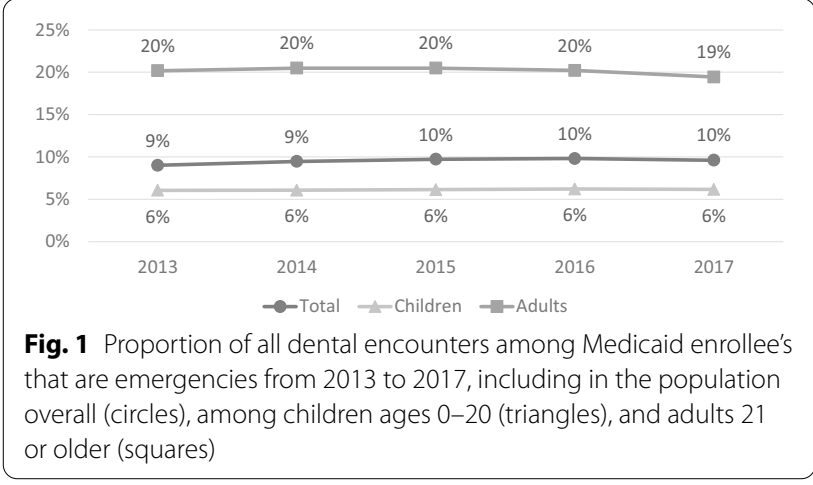

\section{Results}

The analysis is derived from data of over 12 million Medicaid enrolled members in 2016 (with enrollment totals ranging from 11 million in 2013 to 13.5 million in 2017), of whom 3.9 million visited a dentist and 649,442 had an emergency dental visit (Table 1). The demographic characteristics of the data used in this analysis are similar to the results of a nationally representative sample of Medicaid enrollees, with the exception of racial characteristics, where our sample had more Blacks and fewer Hispanics than the nation as a whole.

Out of all dental encounters in 2016, $10 \%$ were emergency dental encounters. When assessed by age group, emergency encounters accounted for $6 \%$ of total encounters for children and $20 \%$ for adult encounters (Fig. 1).

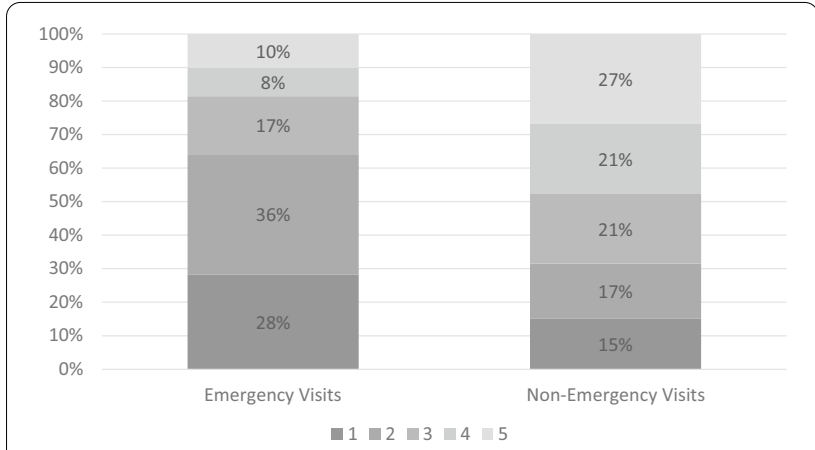

Fig. 2 Number of dental procedures performed during a dental visit, comparing emergency visits on the left and non-emergency visits on the right

These numbers were nearly identical from 2013 to 2017 (Fig. 1), demonstrating stability in these estimates over time. $28 \%$ of emergency dental encounters had no other procedure performed during those encounters, other than the emergency visit code, while another 36\% had only one additional dental procedure performed during the visit (Fig. 2). Most additional procedures performed during an emergency dental visit were related to radiographs or intraoral images, comprising $61 \%$ of all procedures on children and $68 \%$ of all services performed on adults. The next most common procedure category was oral surgeries, predominantly extractions, comprising $12 \%$ of all services performed on children and $17 \%$ of all 
services performed on adults (See Table 2, See Additional file 1: Table $\mathrm{S} 1$ for the most common CDT codes).

$36 \%$ of adult dental emergency visits had a prescription associated with them and 19\% of visits by adults were associated with an opioid prescription. Among children, $15 \%$ of visits had an associated prescription and $4 \%$ had an associated opioid prescription. The most common prescription category prescribed for all emergency dental visits was Opioids at $28 \%$ of all prescriptions. The next most common prescription category was Penicillin with $27 \%$ followed by NSAIDS with $13 \%$ (Fig. 3).

Of all patients with an emergency dental visit in 2016, $78 \%$ returned to the dentists within 365 days of their first emergency dental visit. Among those patients that return, we see significantly fewer imaging procedures as a percent of total and more preventive and treatment (Table 2, Additional file 1: Table S2). Among children, imaging as a percent of total, dropped from 60 to $23 \%$ between their first emergency dental visit and their next follow-up. For adults, imaging dropped from 68 to $28 \%$. Preventive services, in children, went up from 5.4 to 28.2\%. Restorations (both Minor and Major), as a percent of total, went up from 10.9 to $14.5 \%$ in children and from 7.8 to $17.4 \%$ in adults.

Categorizing return visits, $19 \%$ were for another emergency dental visit, $43 \%$ were for dental treatment, such as a restorative or surgical procedure, and $39 \%$ were for routine dental care, such as preventive care (Table 3 ). Patients are most likely to return for a follow-up within 15 days of an emergency dental visit if the follow-up visit is a treatment visit, as $44 \%$ of treatment visits occurred in this time frame. Meanwhile, $32 \%$ of return visits that were also emergency visits occurred within 15 days. Only $16 \%$ of return routine visits occurred within 15 days, and

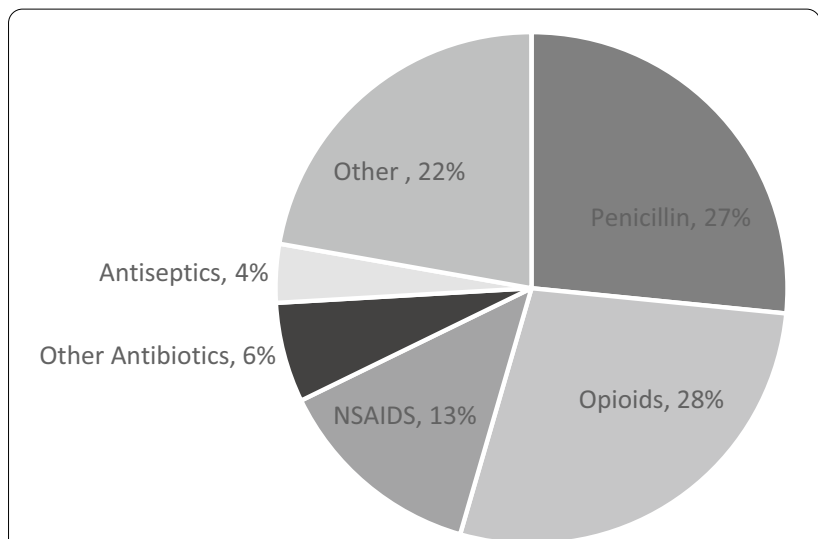

Fig. 3 Type of medication prescribed on the same day as an emergency dental visit, defined by therapeutic class of drug

nearly half occurred 90 or more days after the dental emergency.

Emergency dental visits at a dentist's office can lead to emergency room (ER) visits for dental conditions, as patients are more likely to visit the ER for a dental condition within 15 days of a dental visit if the dental visit was classified as an emergency dental visit. $1.7 \%$ of adult emergency dental visits resulted in an ER visit for a dental condition within 15 days ( $0.6 \%$ in children), compared to $0.6 \%$ for non-Emergency adult dental visits $(0.1 \%$ in children). The emergency dental to ER pathway can lead to a vicious cycle of visits, likely due to unresolved issues, as $3.4 \%$ of emergency dental visits among adults ( $1 \%$ among children) occur within 15 days of an ER visit for a dental condition. Additionally, half of patients who have both an emergency dental visit and an ER visit for a dental condition have 2 or more emergency dental visits

Table 2 Procedure code groupings

\begin{tabular}{|c|c|c|c|c|}
\hline \multirow[t]{2}{*}{ Grouping } & \multicolumn{2}{|l|}{ Children } & \multicolumn{2}{|l|}{ Adults } \\
\hline & Emergency visit (\%) & $\begin{array}{l}\text { Visit after emergency visit } \\
\text { (\%) }\end{array}$ & Emergency visit (\%) & $\begin{array}{l}\text { Visit } \\
\text { after emergency } \\
\text { visit (\%) }\end{array}$ \\
\hline Imaging & 61 & 23 & 68 & 28 \\
\hline Oral surgery & 12 & 9 & 17 & 23 \\
\hline Anesthesia & 7 & 8 & 2 & 4 \\
\hline Minor restorations & 7 & 9 & 6 & 13 \\
\hline Preventive & 5 & 28 & 1 & 8 \\
\hline Major restorations/endodontics & 4 & 6 & 2 & 4 \\
\hline Adjunctive general & 2 & 3 & 2 & 2 \\
\hline Diagnostic & 1 & 15 & 1 & 13 \\
\hline Periodontics & 0.3 & 0.1 & 1 & 2 \\
\hline Prosthodontics/orthodontics & 0.1 & 0.6 & 1 & 3 \\
\hline
\end{tabular}


Table 3 Days to return visit within 365 days after emergency visit, by visit type

\begin{tabular}{lccc}
\hline & $\begin{array}{l}\text { Another emergency visit (19\% of return } \\
\text { visits) (\%) }\end{array}$ & Treatment visit (43\% of return visits) (\%) & $\begin{array}{l}\text { Routine visit (39\% } \\
\text { of return visits) (\%) }\end{array}$ \\
\hline $1-15$ & 32 & 44 & 16 \\
$16-30$ & 15 & 20 & 11 \\
$31-60$ & 15 & 17 & 14 \\
$61-90$ & 9 & 7 & 11 \\
$91-180$ & 15 & 4 & 27 \\
$181-365$ & 14 & 100 & 20 \\
Total & 100 & 400 \\
\hline
\end{tabular}

within 365 days, while slightly more than half have 2 or more ER visits for dental conditions within 365 days.

\section{Discussion}

Dental emergency visits tend to be evaluative in nature or are associated with prescriptions for pain or infection management, rather than providing definitive treatment, as more than 1 in 4 visits had no other procedure associated with them and, when there are other procedures, they tend to be for imaging and other non-definitive treatments. Dental emergency visits, however, are often a pathway to more definitive dental treatment, with $65 \%$ of those who return for dental treatment coming back within 30 days. This finding is in line with contemporary research during the COVID-19 pandemic in the UK, which found that $65 \%$ of urgent dental visits lead to definitive dental treatment and that emergency dental visits can effectively triage patients into needed clinical care [15].

Emergency dental visits, alone, are not enough for keeping people out of the ER for dental conditions, as about $2 \%$ of adults who have an emergency dental visit end up going to a hospital emergency room within 15 days. Rather, a full range of preventive and dental treatments are needed, as emergency dental visits are more often associated with ER visits than non-emergency visits and there can be a vicious cycle of emergency dental and ER visits for dental conditions. Among those enrolled in Medicaid, there is a great deal of stability over time in the rate of emergency dental visits with about $10 \%$ of dental encounters being on an emergency basis.

Efforts to stem the spread of SARS-CoV-2 and COVID19 led to restricting the provision of dental care to dental emergencies. Recommendations to limit routine dental care by the WHO were adopted by many countries [16]. While the response by regulatory and governing bodies has varied, much of the initial focus was on reducing viral spread, ensuring patient and provider safety and allowing time for updates to infection control policies and practice through limiting dental services [17-22].
In the United States of America, the response to COVID-19 from the Centers for Disease Control and Prevention (CDC), a national public health institute in the United States, along with the nation's largest dental association, the American Dental Association (ADA) included initial guidance which encouraged limiting dental care to urgent or emergent treatment [23, 24]. ADA interim guidance on returning to provide non-emergent care urged that treatment should be decided on patient or community risk of COVID-19, clinical risks associated with aerosol generating procedures, and the availability of personal protective equipment[25].

Dental professionals are at considerably high risk of exposure to pathogenic microorganisms that infect the oral cavity and respiratory tract due to the nature of the dental care setting and procedures, which involve a faceto-face proximity between patient and provider, handling of high-speed handpieces, and exposure to saliva, blood, and other body fluids [26-29]. The COVID-19 crisis and the resulting dental service restrictions presented providers, payers, and patients with an unprecedented challenge, and determining the full impact on overall oral health and long-term changes in demand for services will be difficult to predict. These reductions in dental services, even for a short period of time, will have significant impact on the oral health of Americans. Recent analysis has shown that $92 \%$ of families in poverty or low incomes have unmet dental needs [30]. These families rely on public insurance programs and access to low-cost or free dental services to address their needs. Given the great burden of dental disease in these populations, limitations on scope of service and dental office closures across the country have had a disproportionate impact on individuals experiencing poverty, the uninsured, and individuals who participate in United States government-sponsored programs such as Medicaid, which helps cover health care costs for low-income Americans, generally under the age of 65 [31].

There are several limitations to this study. While the dataset used is a large database with a population that is 
consistent with the Medicaid population as whole, it is not randomly generated sample of all Medicaid claims in the United States and so should not be assumed to be definitively generalizable. Additionally, while we focused on the Medicaid population to both the lack of information on this population and their unique vulnerability to untreated dental conditions, the Medicaid population is not like the population as a whole in the United States. Due to the consistency in trends over time, this study focuses on a single year of data for the bulk of the analysis and so cannot definitely state that the findings hold across all years of data nor that the patterns will hold across time. Regardless of the limitations, we believe that the findings reported here, including the stability of emergency dental visits over time, the nature of those visits as predominately evaluative in nature, and the outcomes of those visits for patients as often resulting in definitive treatment, but that they can lead to a cycle of emergency dental and hospital utilization for a small subset of patient provide useful information that can be built on by future work and used to make decisions in the current environment.

\section{Conclusion}

Given what is known about the infection pathways of COVID-19 and with a general understanding of emergency dental visits in the dental setting, providers can effectively plan and determine how to provide essential care to patients while protecting themselves, staff, and the individuals who are seen for treatment. The provision of care under the threat of COVID-19 or other air-borne infections will require the adoption of new techniques for infection control in dental settings. Additional emphasis on the reduction of aerosols may result in increased usage of non-aerosol generating treatments for carious lesions, such as silver diamine fluoride, temporary restorations with glass ionomers, or atraumatic restorative treatments [32-34]. Providers should be prepared for evolving guidance and continued adjustments to the way they practice, as evidence-based research is established for COVID-19 and dental treatment.

Significant efforts have been made in providing and examining infection control and clinical management of dental emergencies. While many practices have reopened with increased infection control procedures, the pandemic is not well-controlled in many regions of the United States and there may be ongoing delays in people receiving dental care or even additional shutdowns of dental care [35]. Remote triage tools, such as teledentistry can assist in keeping providers and patients engaged and communicating effectively throughout the changes in operations and may be an essential tool in the transition to a new era in dentistry, assuming that regulatory and financial barriers to implementation are addressed in an expedient manner [36]. Through teledentistry and in collaboration with patients, providers can engage in more patient outreach, reinforce healthy behaviors, provide education, and explore minimally invasive treatment options without being in physical proximity. Additionally, providers who connect with patients via telehealth can triage and direct care to appropriate settings, maximizing the productive use of patient time in the operatory while reducing exposure risk. As a result of the COVID19 pandemic, providers have the opportunity to refocus care delivery to be more agile in responding to future closures, reducing the demand for emergency services through pathway redesign, and moving from a "drill first" focus to prevention-focused built around the most minimally invasive procedures possible.

\section{Supplementary information}

Supplementary information accompanies this paper at https://doi. org/10.1186/s12903-020-01345-7.

Additional file 1. Top 20 Procedure Codes During Emergency Visits and Visit after an Emergency Visit.

\section{Abbreviations}

ADA: American Dental Association; ASTDD: Association of State and Territorial Dental Directors; CDC: Centers for Disease Control and Prevention; CDT: Code on Dental Procedures and Nomenclature; CMS: Centers for Medicare and Medicaid Services; COVID-19: Coronavirus disease 2019; ER: Emergency room; FQHC: Federally Qualified Health Centers; HRSA: Health Resources and Services Administration; ICD: International Statistical Classification of Diseases and Related Health Problems; MUA: Medically underserved areas.

\section{Acknowledgements}

The authors would like to acknowledge Dr. Julie Hawley for her critical review of the manuscript. The authors would also like to acknowledge Dr. Sean Boynes for his contribution to the conception of the analysis

\section{Authors' contributions}

RF was a major contributor in drafting and revising the manuscript and interpreting the data. $\mathrm{IO}$ contributed to the analysis and interpretation of the data and substantively revised the analysis. MB contributing to the design of the manuscript. SB substantively revised the manuscript and contributed to the interpretation of the data. ET contributed to the conception of the work, the acquisition, analysis, and interpretation of the data, and substantively revised the manuscript. All authors read and approved the final manuscript.

Funding

No external sources of funding were utilized in this manuscript.

\section{Availability of data and materials}

The data that support the findings of this study are available from International Business Machines (IBM), but restrictions apply to the availability of these data, which were used under license for the current study, and so are not publicly available. No administrative permissions are required to use this data, however a licensing fee for use of the data is generally required. Data may be available from the authors upon reasonable request and with permission of IBM.

\section{Ethics approval and consent to participate}

Because this study uses deidentified data with no ability to link to protected health information, this study was ruled exempt from IRB review by Western IRB. 


\section{Consent for publication \\ Not applicable.}

\section{Competing interests}

The authors declare that they have no competing interests.

\section{Author details}

1 DentaQuest Partnership for Oral Health Advancement, 465 Medford Street, Boston, MA 02129, USA. ${ }^{2}$ DentaQuest, 11100 West Liberty Drive, Milwaukee, WI 53224, USA.

Received: 8 May 2020 Accepted: 22 November 2020

Published online: 04 December 2020

\section{References}

1. Brecher EA, Keels MA, Best AM, Quiñonez RB, Roberts MW. Management of after-hours pediatric dental emergencies among pediatric and general dentists. Pediatr Dent. 2018;40(5):352-8.

2. Meyer BD, Lee JY, Lampiris LN, Mihas P, Vossers S, Divaris K. "They told me to take him somewhere else": Caregivers' experiences seeking emergency dental care for their children. Pediatr Dent. 2017;39(3):209-14.

3. Huang SM, Huang JY, Yu HC, Su NY, Chang YC. Trends, demographics, and conditions of emergency dental visits in Taiwan 1997-2013: a nationwide population-based retrospective study. J Formos Med Assoc. 2019;118(2):582-7.

4. Huang JY, Yu HC, Chen YT, Chiu YW, Huang SM, Chang YC. Analysis of emergency dental revisits in Taiwan (1999-2012) from Taiwanese national health insurance research database (NHIRD). J Dent Sci. 2019:14(4):395-400.

5. Okunseri C. Limiting dental benefits may lead to hospital emergency department visits for nontraumatic dental conditions. J Evid Based Dent Pract. 2018;18(2):185-6.

6. Sun BC, Chi DL, Schwarz E, Milgrom P, Yagapen A, Malveau S, et al. Emergency department visits for nontraumatic dental problems: a mixedmethods study. Am J Public Health. 2015;105(5):947-55.

7. Emergency Department and Inpatient Hospitalization for Non-Traumatic Dental Conditions in Texas. Austin, TX: Texas Health Institute; 2018.

8. Hospital Visits for Dental Conditions in Maryland. Westborough, MA DentaQuest Institute.

9. Hammel JM, Fischel J. Dental emergencies. Emerg Med Clin North Am. 2019;37(1):81-93.

10. Guo H, et al. The impact of the COVID-19 epidemic on the utilization of emergency dental services. J Dent Sci. 2020. https://doi.org/10.1016/j. jds.2020.02.002

11. Tramini P, Al Qadi Nassar B, Valcarcel J, Gibert P. Factors associated with the use of emergency dental care facilities in a French public hospital. Spec Care Dent. 2010;30(2):66-71.

12. Roberts RM, Bohm MK, Bartoces MG, Fleming-Dutra KE, Hicks LA, Chalmers NI. Antibiotic and opioid prescribing for dental-related conditions in emergency departments: United States, 2012 through 2014. J Am Dent Assoc. 2020;151(3):174-81.e1.

13. International Business Machines. IBM Watson Medicaid Marketscan Database. 2013-2017.

14. IBM Watson Health, 2019. IBM MarketScan Research Databases for life sciences researchers.

15. Carter E, Currie C, Asuni A, Goldsmith R, Toon G, Horridge C, Simpson S, Donnell C, Greenwood M, Walton G, Cole B. The first 6 weeks: setting up a UK urgent dental care centre during the COVID-19 pandemic. medRxiv. 2020.

16. Considerations for the provision of essential oral health services in the context of COVID19: World Health Organization; 2020. https://apps.who. int/iris/bitstream/handle/10665/333625/WHO-2019-nCoV-Oral healt h-2020.1-eng.pdf. Accessed 5 Apr 2020.
17. Meng L, Hua F, Bian Z. Coronavirus disease 2019 (COVID-19): emerging and future challenges for dental and oral medicine. J Dent Res. 2020:99(5):481-7.

18. Noh K, Loke J, Kim K. Could we have prevented all this? A comparison of the British and South Korean primary dental care response to COVID-19. Br Dent J. 2020;228(12):916-8.

19. Rupf S, Hannig M. Changes of the patient management in dentistry during the pandemic caused by the SARS-Coronavirus 2-initial perspectives of a clinic of operative dentistry in Europe. Clin Oral Invest. 2020;24(7):2537-9.

20. Singh R, Dhoble A. Advisory on Corona: Indian Dental Association; 2020.

21. Guidelines for oral health services at COVID-19 Alert Level 4: Dental Council New Zealand; 2020

22. Issue 3, Preparedness letter for primary dental care-25 March 2020: NHS England; 2020.

23. ADA Interim Guidance for Minimizing Risk of COVID-19 Transmission: American Denal Association; 2020. https://www.ada.org/ /media/CPS/ Files/COVID/ADA_COVID_Int_Guidance_Treat_Pts.pdf. Accessed 5 Apr 2020.

24. Non-Emergent, Elective Medical Services, and Treatment Recommendations. Centers for Medicare \& Medicaid Services; 2020. https://www.cms. gov/files/document/cms-non-emergent-elective-medical-recommenda tions.pdf. Accessed 5 Apr 2020.

25. Return to Work Interim Guidance Toolkit Chicago, IL: American Dental Association; 2020. https://success.ada.org/ /media/CPS/Files/Open\%20 Files/ADA_Return_to_Work_Toolkit.pdf. Accessed 5 Apr 2020

26. Guidelines for oral health services at COVID-19 Alert Level 4. Dental Council New Zealand; 2020

27. Peng $X, X u X$, Li Y, Cheng L, Zhou $X$, Ren B. Transmission routes of 2019nCoV and controls in dental practice. Int J Oral Sci. 2020;12(1):9.

28. Chen J. Pathogenicity and transmissibility of 2019-nCoV-a quick overview and comparison with other emerging viruses. Microbes Infect. 2020;22(2):69-71.

29. Cleveland JL, Gray SK, Harte JA, Robison VA, Moorman AC, Gooch BF. Transmission of blood-borne pathogens in US dental health care settings: 2016 update. J Am Dent Assoc. 2016:147(9):729-38.

30. Halasa-Rappel YA, Brow A, Frantsve-Hawley J, Tranby E. Poor families spent 10 times more of their income on dental care than wealthier families. Boston: DentaQuest Partnership for Oral Health Advancement; 2019.

31. Rozier RG, White BA, Slade GD. Trends in oral diseases in the U.S. population. J Dent Educ. 2017;81(8):eS97-109.

32. Galui S, Pal S, Pabale S, Saha S, Sarkar S. Stretching new boundaries of caries prevention with silver diamine fluoride: a review of literature. Int J Pedod Rehabil. 2018:3:1.

33. Gurgan S, Kutuk ZB, Ergin E, Oztas SS, Cakir FY. Clinical performance of a glass ionomer restorative system: a 6-year evaluation. Clin Oral Investig. 2017;21(7):2335-43.

34. de Amorim RG, Frencken JE, Raggio DP, Chen X, Hu X, Leal SC. Survival percentages of atraumatic restorative treatment (ART) restorations and sealants in posterior teeth: an updated systematic review and metaanalysis. Clin Oral Investig. 2018:22(8):2703-25.

35. Tranby E, Thakkar M, Jacob M, Frantsve-Hawley J. Dental care's new normal: provider survey reveals the need to adapt and redesign. Boston: DentaQuest Partnership for Oral Health Advancement; 2020.

36. Fast-Track to Teledentistry. Removing barriers to care while maximizing overall health. Boston: DentaQuestPartnership for Oral health Avancement; 2020.

\section{Publisher's Note}

Springer Nature remains neutral with regard to jurisdictional claims in published maps and institutional affiliations. 